\title{
CHEMISTRY OF PLANT GROWTH SUBSTANCES
}

CHEMICAL structure in relation to plant growth-
held in Sweden in the Botanical Department of
the University of Lund during August 10-14. The
meeting was arranged by Prof. H. Burström (Lund),
Dr. H. Veldstra (Amsterdam) and Prof. R. L. Wain
(London), and participation was restricted to active
workers in this field. Some forty chemists and
biologists from eight countries were present.

Dr. Veldstra, who introduced the first session on "Structure and Activity", gave a review of published work in this field and an account of his own recent investigations. In particular, he showed that compounds like 1-naphthylmethanesulphonic and 1-naphthalenesulphonic acids can show growth-regulating activity at higher concentrations $\left(10^{-3}-10^{-2} M\right)$. In view of the high dissociation constants of these acids, it would appear that the anion is active in promoting the growth response. Dr. C. Hansch (Claremont, U.S.A.) discussed the evidence for the reaction of a growth substance with a plant substrate. His suggestions that, within the plant, a nuclear chlorine atom can be removed from certain chlorinated benzoic acids and that a methyl group might be eliminated, for example, from the growth substance 3-chloro-2 : 6-dimethylbenzoic acid, promoted a lively discussion. In assessing growth-regulating activity Dr. Hansch rates a compound as active if it promotes a 5 per cent increase in growth above the controls in the Avena cylinder test. This method of assessment was criticized as one in which synergism may play an important part. In the last paper of this session, Prof. H. Söding (Hamburg) discussed the chemical nature of the growth substance in Avena coleoptiles. In recent years Prof. Söding has made a survey of the data relating to the possible existence of auxins $a$ and $b$, and a re-analysis of the evidence relating to acid and alkali stability and rates of diffusion has led him to conclude that, so far, indole compounds are the only auxins which have been established as occurring naturally within the plant.

The second session was devoted to papers on optical isomerism in relation to growth-regulating activity. Dr. H. Matell (Uppsala) described how he has determined configurations in a series of optically active aryloxyalkylcarboxylic acids by constructing melting-point curves. When the optical isomers of similar aryloxy acids are studied in this way, the curves reveal the formation of quasi-racemates when the two isomers differ in configuration. Dr. B. Aberg (Uppsala) followed with a paper on the physiological effects of these compounds. His results were in agreement with the :dea that activity involves the association of the compound with some enzyme system, certain groupings in the molecule becoming associated with specific receptor groups on the enzyme surface. His work is closely in line with that of Prof. Wain, Prof. Burström and others, and in discussion it was evident that much work on antiauxins and auxin antagonists is now proceeding. This field of investigation was considered further in the third session. Prof. L. J. Audus (London) gave a critical evaluation of the present concepts of auxin antagonists. He made a plea for rigid standardization in the preparation of biological materials and in experimental method, stressing the importance of proper statistical analysis of results. Dr. H. Erdtman (Stockholm) discussed some approaches to the study of anti-auxins, and Dr. C. Mentzer (Lyons) described his investigations on the antagonistic effects of $N$-methyl- $N$-phenylglycine and $N$-methyl- $N$-acetonylaniline on the growth of carrot in tissue culture experiments in vitro.

The fourth session, on the mode of action of growth substances, was opened by Prof. R. L. Wain (London), who discussed the breakdown of certain $\omega$-aryl-and $\omega$-aryloxy-alkylcarboxylic acids in plants. Chemical evidence was presented which indicates that, within the flax plant, phenoxy acids may be degraded by a $\beta$-oxidation mechanism and that with certain chain-lengths $\omega$-oxidation might also occur. These findings were considered in relation to the results obtained by other workers in human and animal metabolism studies. Prof. Wain also indicated the steric considerations which led Toothill to prepare and test the growth-regulating activity of $2: 4$-dichloro-6-fluoro- and 2:4-dibromo-6-fluorophenoxyacetic acids. The high activity found with these compounds was shown to stand in marked contrast to the inactivity of the corresponding $2: 4: 6$-trichloro- and $2: 4: 6$-tribromo-acids. Certain physico-chemical aspects of plant growth. regulating activity were dealt with by Dr. R. C. Brian (Bracknell, Berks), who gave a paper on the interaction of growth substances with monolayers of proteins, lipids and material produced directly from plant tissues. Evidence was presented showing that 2-methyl-4-chlorophenoxyacetic acid is almost completely ionized at the $p H$ occurring within plant cells, where it must therefore exist as the anion. The increase in physiological activity which is observed at lowered $p H$ was attributed to ionization changes in the protein complexes within the plant affecting their interaction with the growth-substance anion. Material obtained from the tissues of different plant species was shown to vary greatly in capacity to adsorb the growth substance; but no general cor. relation between this capacity and susceptibility of the plant to the herbicidal action of the chemical could be drawn. Drs. H. Linser and K. Kaindl (Linz, Austria) discussed the concentration/activity curves of various growth activators and growth inhibitors in the "Avena-Pastentest" and the interpretation of the results. Dr. H. L. Booij (Leyden), in a theoretical contribution on mode of action, directed attention to the similarity between the action of plant growth substances and those of haptens in serological reactions. He pointed out that, in general, both types of compound are water-soluble, have low molecular weights and they may operate as anions. It was suggested that certain enzymes bound to protein within the resting plant cell might be liberated under the action of a growth substance in a manner analogous to that in which a hapten, by associating with a specific antibody, affects the normal antigen-antibody relationship.

The final session, dealing with physiological aspects, was opened by Prof. K. V. Thimann (Harvard), who described work with certain compounds which might arise from the oxidation of 3 -indolylacetic adid. The inactivity of these substances has led him to consider it unlikely that an oxidation mechanism is 
involved in the growth-regulating activity of this acid. In his studies on anti-auxins, Prof. Thimann has found that, under certain conditions, 3-methylindole can depress the activity of 3 -indolylacetic acid. The effect is not shown with low concentrations of the acid, indicating that competitive antagonism is not involved and that 3-methylindole is not an antiauxin. Prof. Thimann has concluded from further experiments that $2: 4$-dichloroanisole, claimed to be an anti-auxin by Bonner, is likewise a growth inhibitor only. Studies with 3 -indolylacetonitrile were also described by Prof. Thimann. Evidence was presented which indicates that, in tests where the nitrile shows activity, such activity depends upon its conversion to 3 -indolylacetic acid within the plant tissue. Prof. H. Burström (Lund), in a comprehensive physiological paper, made an attempt to define growth activity. In particular, he dealt with auxin and anti-auxin activity, the type of antagonism shown only by external growth substances, the disturbance of geotropic responses, and toxicity. The paper, which was illustrated throughout by detailed reference to the growth effects obtained with specific compounds, focused attention on the complex interplay of physiological processes which may be concerned in promoting the various types of growth response.

R. L. WAIN

\section{CHEMICAL PROCESSES AND CHEMICAL ENGINEERING TECHNIQUES}

$\mathrm{T}$ HE first chemical plant exhibition to be held in Great Britain since 1936 was housed at Olympia, London, during September 3-17 in conjunction with the annual engineering, marine and welding exhibitions. The Institution of Chemical Engineers, together with the Chemical Engineering Group of the Society of Chemical Industry, marked the occasion by holding a chemical engineering conference during the five days commencing September 7.

The meetings were avowedly general in nature, being designed to support the plant exhibition, and the theme of the entire conference could be described as one of 'materials and methods'. Perhaps the focal point of the meetings was that held under the chairmanship of Mr. Stanley Robson, at which the implications of recent reports upon American practice ("Chemical Apparatus in the U.S.A." by the Organization for European Economic Co-operation, and "Heavy Chemicals" by the Anglo-American Council on Productivity) were considered.

Sir Harold Hartley and Mr. J. Grange Moore initiated a wide discussion in which the most preeminent views were that industry needs more chemical engineers, but should also make better use of those it has. Prof. M. B. Donald warned the profession that it would find itself starved of entrants unless something were done to reduce the serious shortage of science teachers in grammar schools.

Around this central theme, covering the whole scope of chemical engineering, other papers were placed dealing with more specific developments in processes and techniques. Among these were contributions dealing with some unusual materials of construction. Thus, carbon and graphite (Mr. A. W. Morrison), tantalum and zirconium (Dr. G. L. Miller) and plastic materials (Mr. Verney Evans) were presented, and their special properties and applica- tions in the chemical ind astries illustrated. Much interest was shown in thesr topics; and the importance of constructional materia s, in that their development is often the limiting fac or in the application of new processes, was stressed by more than one speaker.

The remaining papers presented to the conference dealt with chemical processes and chemical engineering techniques. Two interesting examples of specialist design and operation resulting from the penetration of chemical engineering fundamentals into two specific processes were provided by papers on milk processing plant (Mr. J. E. F. Renton) and formaldehyde production (Mr. K. Nickels).

The increasing use of chlorine throughout the industry made of timely interest a paper on developments in the production of chlorine in mercury cells (Mr. L. R. Thomas), particularly as the author drew many useful comparisons between alternative cell designs. A paper on advances in evaporation, with particular reference to the concentration of heatsensitive liquids (Mr. B. N. Reavell), provided further examples of the application of a general technique to a specific problem. This provoked a lively discussion by its treatment of the economics of multipleeffect evaporation, as well as by a description of lesser-known systems, such as low-temperature elimbing-film machines operating on the heat-pump principle.

Of more general interest was a review of developments in distillation since 1940 (Mr. G. A. Dummett). Although necessarily very compressed, the paper made an assessment of the important progress which has been made during the past thirteen years in fundamental physical data, in theoretical design methods and in practical industrial equipment.

In ranging from the very general comparison of the practice of chemical engineering in Great Britain and the United States to the wide distribution of a particular operation, this brief report discusses in a re-arranged order the papers of the conference and not in the order in which they were read.

T. K. Ross

\section{BRITISH PHARMACEUTICAL CONFERENCE, 1953}

THE ninetieth British Pharmaceutical Conference was opened at Grosvenor House, London, on August 31, and more than six hundred pharmacists attended. The range of topics covered in the scientific sessions illustrates the extensive development of pharmaceutical research.

Fresh interests in the constituents of plants were demonstrated by several workers who have applied new methods of analysis. The quantitative estimation of the ergot alkaloids has been achieved by partition chromatography on cellulose columns, and colorimetric assay of the fractions shows that recoveries of pure individual alkaloids are consistent. The constituents of digitalis tinctures have been investigated, no correlation having been found between the potency of frogs to the glycosides and the intensity of the colour reaction. Other workers have applied paperpartition chromatography to the separation of the tropane alkaloids, their estimations being carried out by use of the Vitali-Morin reaction. The chromatographic method has been particularly useful in estimating the hyoscine and hyoscyamine content of Datura sanguinea. 